\title{
Fast-time STAP Performance in pre and post Range Processing Adaption as applied to Multichannel SAR
}

\author{
Luke Rosenberg*†, Matthew Trinkle*, Doug Gray* \\ *University of Adelaide, Australia \\ $\dagger$ Defence, Science and Technology Organisation, Australia \\ e-mail: lukerdeleceng.adelaide.edu.au
}

\begin{abstract}
Hot-clutter cancellation using fast-time Space Time Adaptive Processing (STAP) can occur either pre or post range processing $(R P)$ and to date, there has not been a direct comparison on which method offers the best results. This paper provides an analytic comparison which is verified with simulation and aims to provide insight into the location of the adaptive filter which would provide the best hot-clutter suppression. The covariance models are tested with signal models used in a multichannel Synthetic Aperture Radar (SAR).
\end{abstract}

\section{Introduction}

Forming a SAR image in the presence of non-stationary hot-clutter will result in large regions being distorted and unusable. Fast-time STAP has been shown to be an effective method for suppressing the hot-clutter, while maintaining a coherent SAR image, [1]. The processing chain for a received SAR signal involves basebanding, sampling, RP and image formation. RP for SAR involves decoding the received signal with a Matched Filter (MF) to improve the time/bandwidth product and hence achieve a finer range resolution.

Previous studies on the performance of hot-clutter suppression algorithms have looked at using an adaptive filter with no RP, [2] and also post RP, [3]. The former study derived an analytical model for the pre RP covariance and tested its performance under different simulated topographical conditions, while the latter study derived a post RP covariance model and used a simulated scenario to compare element space and beam space approaches. The only study on fast-time STAP performance for SAR is by Klemm, [4] who looked at simulated data applied pre RP and concluded that firstly, fast-time filtering will degrade SAR resolution by broadening the point spread function mainlobe and increasing its sidelobes and secondly, as range resolution improved, sensitivity to filtering increased.

This paper presents fast-time STAP models for pre and post RP adaptive filters and compares the relative adaptive performance as the number of fast-time taps is increased.

\section{Signal model}

The total received signal at the SAR, $x_{n}(\cdot)$ includes the total ground return, interference from the direct-path and ground reflected path (hot-clutter) and receiver noise. The bistatic jammer model is formed by the superposition of the direct path and $K$ hot-clutter patches within a given area,

$$
z_{n}\left(t_{l}, u\right)=\sum_{k=0}^{K} b_{k} J\left(t_{l}-\bar{\tau}_{n, k}\left(t_{l}, u\right)\right) \exp \left[-j \omega_{c} \bar{\tau}_{n, k}\left(t_{l}, u\right)\right] \exp \left[-j \omega_{d, k} t_{l}\right]
$$

where $\left(t_{l}, u\right)$ represents the $l^{\text {th }}$ fast-time sample within a pulse and the SAR position respectively, $J(\cdot)$ is the jamming signal waveform, $\bar{\tau}_{n, k}(\cdot)$ is the bistatic delay for the $k^{t h}$ patch, $\omega_{d, k}$ is the fasttime doppler frequency and $b_{k}$ is the relative magnitude between the direct-path and hot-clutter signals. The zero index refers to the direct-path with $b_{0}=1$. 
Realisations of the jammer signal $J(\cdot)$ can be generated by an eigen-decomposition of the jammer auto-covariance, $r_{J}(\tau)=\sigma_{J}^{2} \operatorname{sinc}(B \tau)$ with bandwidth, $B$ and power level, $\sigma_{J}^{2}$. The relative scattering magnitude is determined by a physically based model for the multipath scattering, [5]. It uses a rough surface to define the scattering distribution between the SAR and an airborne jammer. The coefficients, $b_{k}=\rho B_{k}$ for $k>1$ are formed with a hot-clutter scaling factor $\rho$, relative to the direct-path and a random amplitude $B_{k}$, determined from the scattering model.

\section{Fast-time STAP}

The focussed output from the fast-time STAP filter is determined by the following convolution over $\tilde{L}$ taps,

$$
\begin{aligned}
x_{\mathrm{fs}}\left(t_{l}, u\right) & =\sum_{q=l}^{l+\tilde{L}-1} \mathbf{H}^{H}\left(t_{l}-t_{q}, u\right) \mathbf{x}\left(t_{q}, u\right) \\
& =\tilde{\mathbf{H}}^{H}(u) \mathbf{X}\left(t_{l}, u\right)
\end{aligned}
$$

where $\tilde{\mathbf{H}}(\cdot)$ is the space/fast-time steering vector and $\mathbf{X}(\cdot)$ is the fast-time received data vector. If there are $N$ antenna elements, the received data signal can be stacked twice with the reference antenna at the centre of the array to give,

$$
\begin{aligned}
\mathbf{x}\left(t_{l}, u\right) & =\left[x_{-(N-1) / 2}\left(t_{l}, u\right), \ldots, x_{(N-1) / 2}\left(t_{l}, u\right)\right]^{T} \in C^{N \times 1} \\
\mathbf{X}\left(t_{l}, u\right) & =\left[\mathbf{x}^{T}\left(t_{l}, u\right), \mathbf{x}^{T}\left(t_{l+1}, u\right), \ldots, \mathbf{x}^{T}\left(t_{l+\tilde{L}-1}, u\right)\right]^{T} \in C^{\tilde{L} N \times 1}
\end{aligned}
$$

with data components for the final $\tilde{L}$ taps set to zero. Fast-time STAP then involves substituting a weight vector for the steering vector in Equation 2. The maximum SINR weight is given by,

$$
\mathbf{W}(u)=\gamma \mathbf{R}_{Z}^{-1}(u) \tilde{\mathbf{H}}(u)
$$

where $\gamma$ is an arbitrary scaling factor and $\mathbf{R}_{Z}(\cdot)$ is the space/fast-time covariance. The form of the pre and post RP space/fast-time steering vectors are based on the spatial and temporal components of the received SAR signal model. The spatial steering model for the $n^{\text {th }}$ channel is given by,

$$
s_{n}(u)=\exp \left[j \frac{\omega_{c}}{c} d_{n} \sin [\theta(u)]\right]
$$

where $\omega_{c}$ is the carrier frequency, $c$ is the speed of light, $d_{n}=n \lambda_{c} / 2$ is the antenna offset from the array phase centre with wavelength $\lambda_{c}$ and $\theta(u)$ is the steering angle relative to the centre of the imaging patch. The spatial steering vector, $\mathbf{s}(u)$ is then formed similarly to Equation 3. The pre RP fast-time steering model is given by the SAR waveform which is a chirp. For the $l^{\text {th }}$ fast-time sample and $q^{\text {th }}$ fast-time tap,

$$
g_{\text {pre }, q}\left(t_{l}\right)=\exp \left[-j B \pi\left(t_{l}-t_{q}\right)+j \alpha\left(t_{l}-t_{q}\right)^{2}\right]
$$

where $B$ is the bandwidth, $2 \alpha$ represents the chirp rate and the fast-time samples occur at $t_{l}=$ $T_{S}+(l-1) \Delta_{t}$ where $T_{S}$ is the pulse collection starting time and $\Delta_{t}$ is the fast-time sampling rate. As the steering vector is a chirp function, it can only provide a partially accurate representation of the target signal within $\tilde{L}$ fast-time taps. The pre RP temporal steering vector shows this,

$$
\mathbf{g}_{\text {pre }}=\left[1, \exp \left[j B \pi \Delta_{t}+j \alpha \Delta_{t}^{2}\right], \ldots, \exp \left[j B \pi(\tilde{L}-1) \Delta_{t}+j \alpha\left((\tilde{L}-1) \Delta_{t}\right)^{2}\right]\right]^{T} \in c^{\tilde{L} \times 1}
$$

and is now a function of the difference between time taps. After RP, the fast-time steering model is represented by,

$$
g_{\text {post }, q}\left(t_{l}\right)=\operatorname{sinc}\left[B\left(t_{l}-t_{q}\right)\right]
$$

which is a decaying function and can therefore be accurately represented by a small number of taps as shown by the post RP temporal steering vector,

$$
\mathbf{g}_{\text {post }}=\left[1, \operatorname{sinc}\left[B \Delta_{t}\right], \ldots, \operatorname{sinc}\left[B(\tilde{L}-1) \Delta_{t}\right]\right]^{T} \in C^{\tilde{L} \times 1}
$$

Consequently better signal detection results will be achieved in $\mathbf{g}_{\text {post }}$ as the signal energy is more concentrated. 
The combined space/fast-time steering vector for each case is then formed by the Kronecker product of the temporal and spatial components to give,

$$
\tilde{\mathbf{H}}_{\text {pre }}(u)=\mathbf{g}_{\text {pre }} \otimes \mathbf{s}(u) \in C^{\tilde{L} N \times 1}, \quad \tilde{\mathbf{H}}_{\text {post }}(u)=\mathbf{g}_{\text {post }} \otimes \mathbf{s}(u) \in C^{\tilde{L} N \times 1}
$$

\section{Covariance Models}

The pre and post interference covariance models are based on the estimated sample matrix form,

$$
\hat{\mathbf{R}}_{Z}(u)=\frac{1}{L_{t}} \sum_{l=1}^{L_{t}} \mathbf{Z}\left(t_{l}, u\right) \mathbf{Z}^{H}\left(t_{l}, u\right) \in c^{\tilde{L} N \times \tilde{L} N}
$$

where $\mathbf{Z}(\cdot)$ is the space/fast-time interference signal formed similarly to Equation 4. Also, as the instantaneous covariance models presented in [2]-[3] are fast-time dependent, the mean estimated covariance models will be used with a minor multiplicative bias determined by the number of averaged fast-time taps, $L_{t}$. Both models also assume that the broadband jammer is a stationary random process within the pulse interval, and therefore the received interference signal before, $z_{n}(\cdot)$ and after RP, $y_{n}(\cdot)$, can be similarly defined. The pre RP covariance is given by,

$$
\begin{aligned}
R_{Z, \mathrm{pre}}\left(u, \zeta, n, n^{\prime}\right)= & E\left\{\frac{1}{L_{t}} \sum_{l=1}^{L_{t}} z_{n}\left(t_{l}, u\right) z_{n^{\prime}}{ }^{*}\left(t_{l}+\zeta, u\right)\right\} \\
= & \sigma_{J}^{2} \sum_{k} \sum_{k^{\prime}} b_{k} b_{k^{\prime}}^{*} \operatorname{sinc}\left[B\left(\zeta+\bar{\tau}_{n, k}-\bar{\tau}_{n^{\prime}, k^{\prime}}\right)\right] \exp \left[-j \omega_{c}\left(\bar{\tau}_{n, k}-\bar{\tau}_{n^{\prime}, k^{\prime}}\right)+j \omega_{d, k^{\prime}} \zeta\right] \\
& \exp \left[-j \Delta \omega_{k, k^{\prime}}\left(T_{S}+0.5\left(L_{t}-1\right) \Delta_{t}\right)\right] \frac{\sin \left[0.5 \Delta \omega_{k, k^{\prime}} L_{t} \Delta_{t}\right]}{L_{t} \sin \left[0.5 \Delta \omega_{k, k^{\prime}} \Delta_{t}\right]}
\end{aligned}
$$

where $\Delta \omega_{k, k^{\prime}}=\omega_{d, k}-\omega_{d, k^{\prime}}$ and $\zeta$ is the delay between two fast-time taps. The post RP covariance requires the range compression to be included in the derivation. At baseband, the MF reference signal is given by $p_{c}^{*}(-t)$, where the SAR waveform is a chirp defined by $p_{c}(t)=$ $b\left(t / T_{p}\right) \exp \left[-j B \pi t+j \alpha t^{2}\right]$ with the chirp rate duration $T_{p}$ defined by $b(t)$, which is unity for $0 \leq t \leq 1$ and zero otherwise. This gives the post RP jammer signal model as,

$$
y_{n}\left(t_{l}, u\right)=\int_{-\infty}^{\infty} z_{n}\left(t^{\prime}, u\right) p_{c}^{*}\left(t^{\prime}-t_{l}\right) d t^{\prime}
$$

The post RP covariance model is then given by,

$$
\begin{aligned}
R_{Z, \text { post }}\left(u, \zeta, n, n^{\prime}\right)= & E\left\{\frac{1}{L_{t}} \sum_{l=1}^{L_{t}} y_{n}\left(t_{l}, u\right) y_{n^{\prime}}{ }^{*}\left(t_{l}+\zeta, u\right)\right\} \\
= & \sigma_{J}^{2} \sum_{k} \sum_{k^{\prime}} b_{k} b_{k^{\prime}}^{*} \operatorname{sinc}\left[B \zeta^{\prime}\left(1-\zeta^{\prime} / T_{p}\right)-\Delta f_{k, k^{\prime}}\left(T_{p}-\zeta^{\prime}\right)\right] \\
& \exp \left[-j \omega_{c}\left(\bar{\tau}_{n, k}-\bar{\tau}_{n^{\prime}, k^{\prime}}\right)+j \omega_{d, k^{\prime}} \zeta^{\prime}\right] \\
& \exp \left[-j \Delta \omega_{k, k^{\prime}}\left(T_{s}+0.5\left(T_{p}-\zeta^{\prime}+\left(L_{t}-1\right) \Delta_{t}\right)\right)\right] \frac{\sin \left[0.5 \Delta \omega_{k, k^{\prime}} L_{t} \Delta_{t}\right]}{L_{t} \sin \left[0.5 \Delta \omega_{k, k^{\prime}} \Delta_{t}\right]}
\end{aligned}
$$

where $\zeta^{\prime}=\zeta-\bar{\tau}_{n, k}+\bar{\tau}_{n^{\prime}, k^{\prime}}$ and $\Delta f_{k, k^{\prime}}=0.5 \Delta \omega_{k, k^{\prime}} / \pi$. This model is very similar to the pre RP case with the sinc function containing the relative temporal delays and the correlation variable. There are now however extra terms related to the pulse width and the differential doppler. These differences are also present in the two exponential terms and will cause only a very small difference in the overall model. These models are used to form covariance matrices, $\mathbf{R}_{Z \text {,pre }}$ and $\mathbf{R}_{Z, \text { post }}$ by varying the spatial channel indices, $n, n^{\prime}$ and the fast-time delay, $\zeta$. 


\section{Simulated Results}

The SINR loss is a common method of comparing adaptive performance and is defined by SINR divided by the interference free Signal to Noise Ratio (SNR) and is a metric between 0 and 1 .

$$
L_{\mathrm{SINR}}(\theta(u))=\frac{\tilde{\mathbf{H}}^{H}(u)\left[\mathbf{R}_{Z}+\mathbf{I}_{\tilde{L} N}\right]^{-1}(u) \tilde{\mathbf{H}}(u)}{\tilde{\mathbf{H}}^{H}(u) \tilde{\mathbf{H}}(u)}
$$

To compare the SINR loss, simulation of a single pulse was used to model the covariance matrix in Equation 12 and the same parameters used for the analytic forms in Equations 13 and 15. The simulation is at X-band with $f_{c}=10 \mathrm{GHz}, B=0.3 \mathrm{GHz}, \Delta_{t}=1 / 2 B, T_{p}=28 \mu s$, $\alpha=3.3 \times 10^{15} \mathrm{rad} / \mathrm{s}, \sigma_{J}^{2}=50 \mathrm{~dB}$ and $\rho=0.5$. There are $N=5$ spatial channels, $L=200$ range bins, $K=100$ hot-clutter patches and the covariance matrices are estimated over $L_{t}=3 \tilde{L} N$ range bins. Results are given in Figure 1 for three different fast-time taps where there is a noticeable decrease in SINR loss of approximately $20 \mathrm{~dB}$ due to the extra correlation between the hotclutter scatterers. To analyse this scenario more closely, the minimum point of the SINR loss is measured as the number of fast-time taps is increased. Figure 2 shows a decrease in SINR loss of close to $24 \mathrm{~dB}$ as more taps are used.
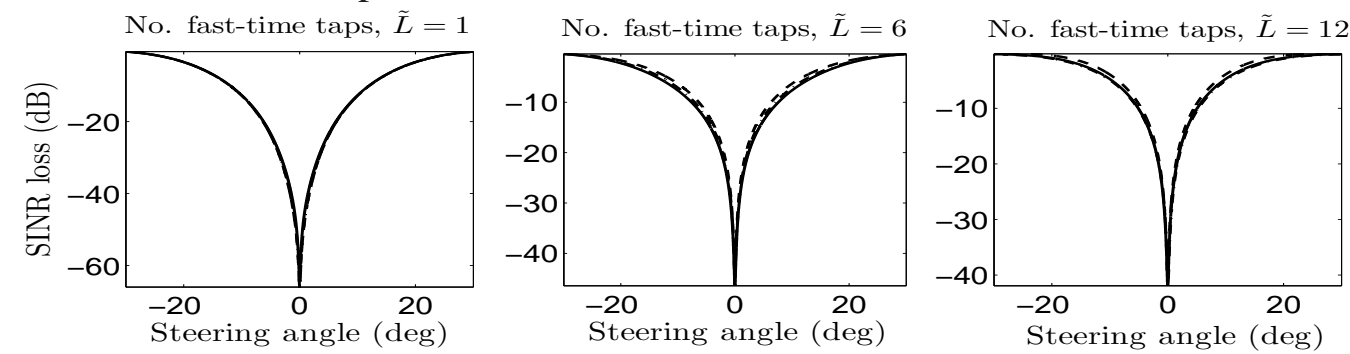

Figure 1: SINR loss with varying $\theta(u)$ for: (-) simulated pre RP, (- -) pre RP, (-.-) post RP

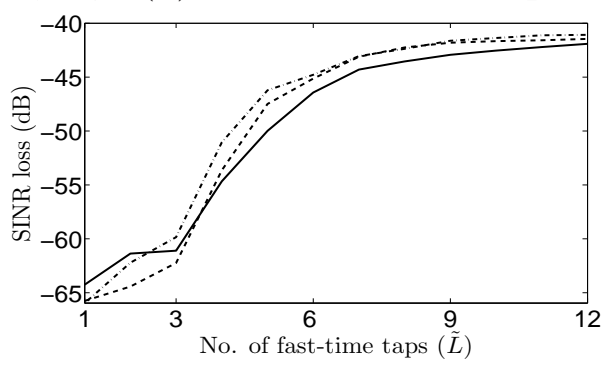

Figure 2: SINR loss with varying $\tilde{L}$ for: (-) simulated pre RP, (- -) pre RP, (-.-) post RP

\section{Conclusion}

In summary, both pre and post RP adaptive filters perform equally well at suppressing hotclutter. However, since the pre RP steering vector only matches a small part of the chirp within the received data and the post RP case matches it almost completely, the latter model is the preferred choice for SAR signal detection.

\section{References:}

[1] L. Rosenberg and D. A. Gray. Fast-time filtering with multichannel SAR. In Adaptive Sensor Array Processing Workshop, June 2005.

[2] P. M. Techau, J. R. Guerci, T. H. Slocumb, and L. J. Griffiths. Performance bounds for hot and cold clutter mitigation. IEEE Transactions on Aerospace and Electronic Systems, 35(4):1253-1265, 1999.

[3] R. L. Fante and J. A. Torres. Cancellation of diffuse jammer multipath by an airborne adaptive radar. IEEE Transactions on Aerospace and Electronic Systems, 31(2):805-820, 1995.

[4] R. Klemm. Principles of Space-Time Adaptive Processing. The Institution of Electrical Engineers, 2002.

[5] P Beckman. The Scattering of Electromagnetic Waves from Rough Surfaces. Pergamon Press Ltd., 1963. 